\title{
Corrigendum: Cytotoxic Effects of Arsenite in Combination With Gamabufotalin Against Human Glioblastoma Cell Lines
}

\author{
Bo Yuan ${ }^{1,2^{*}}$, Kang $X u^{2}$, Ryota Shimada ${ }^{2}$, JingZhe $L i^{3}$, Hideki Hayashi ${ }^{2}$, \\ Mari Okazaki ${ }^{1}$ and Norio Takagi ${ }^{2}$ \\ ${ }^{1}$ Laboratory of Pharmacology, Faculty of Pharmaceutical Sciences, Josai University, Sakado, Japan, 2 Department of Applied \\ Biochemistry, Tokyo University of Pharmacy \& Life Sciences, Hachioji, Japan, ${ }^{3}$ Beijing Key Laboratory of Research of \\ Chinese Medicine on Prevention and Treatment for Major Diseases, Experimental Research Center, China Academy of \\ Chinese Medical Sciences, Beijing, China
}

Keywords: arsenite, gamabufotalin, glioblastoma, cell cycle arrest, p38 MAPK, autophagy, lactate dehydrogenase, combination therapy

\section{A Corrigendum on:}

\section{OPEN ACCESS}

Edited and reviewed by: Nikos Tapinos, Brown University, United States

*Correspondence:

Bo Yuan

yuanbo@josai.ac.jp

Specialty section:

This article was submitted to

Cancer Molecular Targets and Therapeutics,

a section of the journa

Frontiers in Oncology

Received: 17 September 2021 Accepted: 27 September 2021 Published: 08 October 2021

Citation:

Yuan B, Xu K, Shimada R, Li J, Hayashi H, Okazaki M and Takagi N (2021) Corrigendum: Cytotoxic Effects of Arsenite in Combination With Gamabufotalin Against Human

Glioblastoma Cell Lines.

Front. Oncol. 11:778834. doi: 10.3389/fonc.2021.778834
Cytotoxic Effects of Arsenite in Combination With Gamabufotalin Against Human Glioblastoma Cell Lines

by Yuan B, Xu K, Shimada R, Li J, Hayashi H, Okazaki M and Takagi N (2021). Front. Oncol. 11:628914. doi: 10.3389/fonc.2021.628914

In the original article, there was a mistake in the legend for Figures 7A and 8 as published. Betaactin bands in Fig. 4B, 7A and 8 are identical in each figure legend. The correct legend appears below.

FIGURE 7 Prosurvival role of p38 MAPK in the cytotoxicity of U-87 cells treated with the combination of $\mathrm{As}^{\mathrm{III}}$ and gamabufotalin. (A) Following treatment for $48 \mathrm{~h}$ with relatively low concentrations of $A s^{\mathrm{III}}(1,2 \mu \mathrm{M})$ and gamabufotalin $(20,50 \mathrm{nM})$, alone or in combination, the expression profiles of phospho-p38 (p-p38) and p38 were analyzed using western blotting. A representation image of the expression profile of each protein is shown from three independent experiments. The expression levels were expressed as the ratios between each targeted protein and $\beta$-actin protein expression levels, and were compared with those of control group. (B) Following treatment for $48 \mathrm{~h}$ with the combined regimen of $2 \mu \mathrm{MAs}{ }^{\mathrm{III}}+50 \mathrm{nM}$ gamabufotalin; $3.3 \mu \mathrm{M} \mathrm{As}{ }^{\mathrm{III}}+$ $40 \mathrm{nM}$ gamabufotalin, in the presence of absence of $5 \mu \mathrm{M}$ SB203580, a specific inhibitor for p38 MAPK and its negative control SB202474, cell viability was determined by XTT assay. Relative cell viability was calculated as the ratio of the absorbance at $450 \mathrm{~nm}$ of each treatment group against those of the corresponding untreated control group. Data are shown as the means \pm SD $(n \geq 3)$. A p value less than 0.05 was considered as statistically significant $\left({ }^{\$} \mathrm{p}<0.001 ;{ }^{*} \mathrm{p}<0.0001\right.$ vs. control. ${ }^{\$} \mathrm{p}<$ 0.001 vs. As+Gama and As+Gama+SB202474). As, As ${ }^{\mathrm{III}}$; Gama, gamabufotalin; SB203, SB203580; SB202, SB202474. The images of beta-actin are identical to that in Figure 4B since the same experiment samples were used to analyze. 
FIGURE 8 Involvement of autophagic cell death in the cytotoxicity of U-87 cells treated with the combination of $\mathrm{As}^{\mathrm{III}}$ and gamabufotalin. Following treatment for $48 \mathrm{~h}$ with $\mathrm{As}^{\mathrm{III}}$ $(1,2 \mu \mathrm{M})$ and gamabufotalin $(20,50 \mathrm{nM})$, alone or in combination, the expression profiles of LC3 were analyzed using western blotting. A representation image of the expression profile of LC3 is shown from three independent experiments (A). The expression levels were expressed as the ratio between LC3 protein and $\beta$-actin protein expression levels, and were compared with those of control group (B). Cell viability was determined by XTT assay after treatment for $48 \mathrm{~h}$ with the combination of $2 \mu \mathrm{M} \mathrm{As}{ }^{\mathrm{III}}$ and $50 \mathrm{nM}$ gamabufotalin in the presence or absence of wortmannin $(0.25,1 \mu \mathrm{M})(\mathrm{C})$. Data are shown as the means \pm SD $(n \geq 3)$. A p value less than 0.05 was considered as statistically significant $\left({ }^{\int} \mathrm{p}<0.05\right.$ vs. each alone; ${ }^{*} \mathrm{p}<0.05 ;{ }^{*} \mathrm{p}<0.0001$ vs. control; ${ }^{\dagger} \mathrm{p}<0.05 ;{ }^{*} \mathrm{p}<0.0001$ vs. As+Gama). As, As ${ }^{\mathrm{III}}$; Gama, gamabufotalin. The images of beta- actin are identical to that in Figure 4B since the same experiment samples were used to analyze.

The authors apologize for this error and state that this does not change the scientific conclusions of the article in any way. The original article has been updated.

Publisher's Note: All claims expressed in this article are solely those of the authors and do not necessarily represent those of their affiliated organizations, or those of the publisher, the editors and the reviewers. Any product that may be evaluated in this article, or claim that may be made by its manufacturer, is not guaranteed or endorsed by the publisher.

Copyright (c) 2021 Yuan, Xu, Shimada, Li, Hayashi, Okazaki and Takagi. This is an open-access article distributed under the terms of the Creative Commons Attribution License (CC BY). The use, distribution or reproduction in other forums is permitted, provided the original author(s) and the copyright owner(s) are credited and that the original publication in this journal is cited, in accordance with accepted academic practice. No use, distribution or reproduction is permitted which does not comply with these terms. 